\title{
Greens Functions for the Wave Equation
}

\author{
Alex H. Barnett
}

December 28, 2006

\begin{abstract}
I gather together known results on fundamental solutions to the wave equation in free space, and Greens functions in tori, boxes, and other domains. From this the corresponding fundamental solutions for the Helmholtz equation are derived, and, for the $2 \mathrm{D}$ case the semiclassical approximation interpreted back in the time-domain. Utility: scarring via time-dependent propagation in cavities; Math 46 course ideas.
\end{abstract}

\section{Introduction}

The homogeneous wave equation in a domain $\Omega \subset \mathbb{R}^{d}$ with initial conditions is

$$
\begin{array}{rlrl}
u_{t t}-\Delta u & =0 & \text { in } \Omega \times(0, \infty) & \\
u(x, 0) & =f(x) \quad x \in \Omega \quad \text { initial displacement data } \\
u_{t}(x, 0) & =g(x) \quad x \in \Omega \quad \text { initial velocity data }
\end{array}
$$

If $\Omega$ has boundary then we have boundary conditions such as

$$
u=0 \quad \text { on } \partial \Omega \times(0, \infty)
$$

which corresponds to sound-soft walls. We won't consider mixed, time-dependent, or inhomogeneous boundary conditions. Notation: we use plain letters $x$, etc, to indicate points in $\mathbb{R}^{d}$.

We seek the time-dependent Greens function $G_{t}(x, y)$ (where the subscript indicates time as a parameter) which gives the solution at any future time,

$$
u(x, t)=\int_{\Omega} \partial_{t} G_{t}(x, y) f(y) d y+\int_{\Omega} G_{t}(x, y) g(y) d y
$$

Note $G$ depends on $\Omega$. When $\Omega=\mathbb{R}^{d}$ we will use the symbol $W$ instead of $G$ which indicates a fundamental solution. Also by Duhamel's principle the same Greens function may be used to write the solution for the inhomogeneous wave equation, namely replacing (10) by $u_{t t}-\Delta u=h$ where $h$ is a source function on $\Omega \times(0, \infty)$. The solution (5) then has the added 'volume' term

$$
\int_{\Omega} \int_{0}^{t} G_{t-s}(x, y) h(y, s) d s d y
$$


Deriving this required rewriting the wave equation as a first-order system and is explained well by Ch. 5 of [1]. If this term is to be written as a convolution in time, then $G$ must be defined for all real $t$ by setting $G$ to zero for $t<0$ (causality). Then the time-dependent Greens function satisfies

$$
\left(\partial_{t t}-\Delta_{x}\right) G_{t}(x, y)=\delta(x-y) \delta(t), \quad x, y \in \Omega
$$

in the sense of distributions. Note boundary conditions and domain are constant in time, so only time differences $t$ need be considered rather than absolute times.

\section{$2 \quad$ Free space $\mathbb{R}^{d}$}

Our Fourier transform convention is

$$
\hat{u}(k)=\int e^{i k \cdot x} u(x) d x ; \quad u(x)=\frac{1}{(2 \pi)^{d}} \int e^{-i k \cdot x} \hat{u}(k) d k
$$

where integrals are over $\mathbb{R}^{d}$.

Then (11) becomes at each fixed $k$, the ODE

$$
\hat{u}_{t} t(k, t)=-|k|^{2} \hat{u}(k, t)
$$

whose solution is found for $t>0$ by matching the initial conditions,

$$
\hat{u}(k, t)=\hat{f}(k) \cos |k| t+\hat{g}(k) \frac{\sin |k| t}{|k|} .
$$

In the case $k=0$ the limit $k \rightarrow 0$ of the above should be taken. Back in real space, the above products become convolutions over $\mathbb{R}^{d}$, according to the convolution theorem which is with our convention

$$
\hat{u} \hat{v}=\widehat{u * v} ; \quad \widehat{u v}=\frac{1}{(2 \pi)^{d}}(\hat{u} * \hat{v}) .
$$

Returning to real space the solution is then

$$
u(\cdot, t)=\partial_{t} W_{t} * f+W_{t} * g
$$

where the fundamental solution is given by the inverse Fourier transform

$$
W_{t}(x)=\frac{1}{(2 \pi)^{d}} \int e^{-i k \cdot x} \frac{\sin |k| t}{|k|} d k
$$

Notation: subscript $t$ here indicates $W_{t}(\cdot)$ is a function on $\mathbb{R}^{d}$ which has $t$ as a parameter. We now evaluate this integral directly for particular dimensions $d$.

As an aside, note that [5] (for whom the above is equation [27]) performs this integral by analytic continuation $(y \rightarrow i y)$ from the Poisson kernel $e^{-y \sqrt{-\Delta}}$, then the 'subordination identity' which allows $e^{-y \sqrt{-\Delta}}$ to be written as a weighted 
$t$-integral of $e^{t \Delta}$, based on the heat kernel form. This gives the nice result for all $d$,

$$
W_{t}(x)=\lim _{\epsilon \rightarrow 0^{+}} \frac{C_{d}}{d-1} \operatorname{Im}\left[|x|^{2}-(t-i \epsilon)^{2}\right]^{-(d-1) / 2}
$$

where $C_{d}=\pi^{-(d+1) / 2} \Gamma((d+1) / 2)$ appears to be 2 divided by the surface area of a $(d+1)$-dimensional hypersphere. This is in Taylor's book on PDEs, volume 1.

$d=3$ Writing $d k=k^{2} d k d \mu d \phi$ where $\mu=\cos \theta$, and $(k, \theta, \phi)$ is the wavenumber in spherical polar coordinates, and $r=|x|$, then (13) becomes

$$
\begin{aligned}
W_{t}(x) & =\frac{1}{(2 \pi)^{3}} \int_{0}^{2 \pi} d \phi \int_{0}^{\infty} k^{2} d k \frac{\sin k t}{k} \int_{-1}^{1} e^{-i k \mu r} d \mu \\
& =-\frac{1}{8 \pi^{2} r} \int_{0}^{\infty}\left[e^{i k(t+r)}+e^{i k(t-r)}\right] d k+\text { c.c. } \\
& =\frac{1}{4 \pi r}[\delta(t-r)-\delta(t+r)] \\
& =\frac{\delta(t-r)}{4 \pi r}
\end{aligned}
$$

The $k$-integral was performed by recognising that $2 \pi \delta(x)=\int e^{i k x} d k$ which follows from (8) and is a special case of (30). Notice the second delta vanishes since we consider the causal case $t>0$. Note this differs from the 'method of spherical means' [1] which converts the $d=3$ (or higher odd number) case to a 1D PDE, the Euler-Poisson-Darboux equation, which is satisfied by the integral of $u$ over an expanding sphere. That avoids Fourier methods altogether.

$d=2$ Consider $\tilde{u}$ satisfying the wave equation in $\mathbb{R}^{3}$, launched with initial conditions invariant in the 3 -direction:

$$
\begin{aligned}
\tilde{u}\left(x_{1}, x_{2}, x_{3}, 0\right) & =\tilde{f}\left(x_{1}, x_{2}, x_{3}\right)=f\left(x_{1}, x_{2}\right), \\
\tilde{u}_{t}\left(x_{1}, x_{2}, x_{3}, 0\right) & =\tilde{g}\left(x_{1}, x_{2}, x_{3}\right)=g\left(x_{1}, x_{2}\right),
\end{aligned}
$$

for all $x_{3}$. Then any slice through $\tilde{u}$ perpendicular to the 3 -direction, for instance $u\left(x_{1}, x_{2}, t\right):=\tilde{u}\left(x_{1}, x_{2}, 0, t\right)$, is a solution to the $2 \mathrm{D}$ wave equation with initial conditions $f$ and $g$. This follows since $\tilde{u}$ remains 3-invariant for all $t>0$, so the 3D $\Delta$ operator acting on it is identical to the $2 \mathrm{D} \Delta$ operator. Considering for now the second term in (12), and using (15), we have for any $x \in \mathbb{R}^{3}$,

$$
\begin{aligned}
\tilde{u}(x, t) & =\frac{1}{4 \pi t} \int_{\partial B_{t}(0)} \tilde{g}(y-x) d y \\
& =\frac{1}{4 \pi t} \int_{B_{t}(0)} g\left(y_{1}-x_{1}, y_{2}-x_{2}\right) \frac{2 t}{\sqrt{t^{2}-|x|^{2}}} d x_{1} d x_{2}
\end{aligned}
$$

where the Jacobean of the projection of the spherical shell onto the 2D ball was used (noting the factor of 2 since there are 2 hemispheres). This Jacobean is 
the same as that from the circle in the plane to a line. (16) is now in the form of a $2 \mathrm{D}$ convolution $u=W_{t} * g$ where

$$
W_{t}(x)=\frac{1}{2 \pi\left(t^{2}-|x|^{2}\right)^{1 / 2}} \quad \text { for }|x|<t, \quad \text { zero otherwise. }
$$

This is Hadamard's method of descent. Notice the $2 \mathrm{D}$ fundamental solution $W_{t}$ is the projection of the $3 \mathrm{D}$ one $\tilde{W}_{t}$,

$$
W_{t}\left(x_{1}, x_{2}\right)=\int_{\mathbb{R}} \tilde{W}_{t}\left(x_{1}, x_{2}, x_{3}\right) d x_{3} .
$$

Equivalently one might start with an initial condition lying solely in the 12 plane (i.e. $\tilde{f}=f\left(x_{1}, x_{2}\right) \delta\left(x_{3}\right)$ etc) then integrate the resulting 3D solution along the 3 -direction to get the $2 \mathrm{D}$ solution.

An alternative based on Folland: the Fourier transform proved in (15) is

$$
\frac{\sin |k| t}{|k|}=\int_{\mathbb{R}^{3}} e^{i k \cdot x} \frac{\delta(t-|x|)}{4 \pi t} d x .
$$

The delta restricts the integral to be over a spherical shell. If we set $k=$ $\left(\kappa_{1}, \kappa_{2}, 0\right)$ where $\kappa$ is a $2 \mathrm{D}$ wavevector, then $e^{-i k \cdot x}$ is invariant in the 3 -direction, and integral may be projected down to the disc of radius $t$ in the 1-2 plane. The Jacobean of the transformation is the same as that from the circle in the plane to a line, and gives (using now $x \in \mathbb{R}^{2}$ )

$$
\frac{\sin |\kappa| t}{|\kappa|}=\int_{\mathbb{R}^{2},|x|<t} e^{i \kappa \cdot x}\left[\frac{1}{4 \pi t} \frac{2 t}{\sqrt{t^{2}-|x|^{2}}}\right] d x .
$$

This now expresses a 2D Fourier transform, thus the term in square brackets must be the fundamental solution.

$d=1$ The above method of descent may be used from $3 \mathrm{D}$ to $1 \mathrm{D}$. Since the projection of a spherical shell of radius $t$ to a line is the constant function $2 \pi t$ within $t$ of the origin and zero outside (a fact known as far back as Archimedes 2200 years ago), this immediately defines the form for $W_{t}$ in $1 \mathrm{D}$,

$$
\begin{aligned}
W_{t}\left(x_{1}\right) & =\int_{\mathbb{R}^{d}} \tilde{W}_{t}\left(x_{1}, x_{2}, x_{3}\right) d x_{2} d x_{3} \\
& =\frac{1}{2} H(t-|x|)
\end{aligned}
$$

where $H(\cdot)$ is the Heaviside step function. Note of course there are more direct and elementary ways to get this result, for instance via factorization of the 1D wave equation operator into two advection operators [1].

\subsection{Summary for free space}

The fields $W_{t}$ due to a velocity point source (and for comparison $\partial_{t} W_{t}$ due to displacement point source), are given by 


\begin{tabular}{l|r|r} 
dimension $d$ & $W_{t}$ (velocity IC) & $\partial_{t} W_{t}$ (displacement IC) \\
\hline 1 & $\frac{1}{2} H(t-|x|)$ & $\frac{1}{2}[\delta(t-x)+\delta(t+x)]$ \\
2 & $\frac{1}{2 \pi}\left(t^{2}-|x|^{2}\right)^{-1 / 2}$ for $|x|<t$ & \\
3 & $\frac{1}{4 \pi \mid x} \delta(t-|x|)$ & \\
\hline
\end{tabular}

So the often-heard statement that both $1 \mathrm{D}$ and $3 \mathrm{D}$ propagate on a spherical shell whereas 2D is the 'odd one out' is misleading: when velocity initial conditions are considered, in 1D propagation is over a ball and in $3 \mathrm{D}$ a shell $(2 \mathrm{D}$ is somewhere inbetween). As $d$ increases, the fundamental solution becomes more singular (less continuous).

Note for all $d$ we have $\int W_{t} d x=t$ so $\int \partial_{t} W_{t} d x=1$. The linear growth of $W_{t}$ follows from that of the $k \rightarrow 0$ limit of the ODE (10); free space has a zero-frequency mode.

\section{$3 \quad$ Torus $\mathbb{T}^{d}$}

Consider $\Omega=\mathbb{T}^{d}=[0,2 \pi)^{d}$, that is, periodic boundary conditions.

The solution may be written in two ways:

1. periodic image sum of fundamental solutions (real-space lattice),

2. spectral sum over eigenmodes of the torus (Fourier-space lattice).

Their equivalence gives the trace formula on the torus (the real-space lattice corresponds to periodic orbits on the torus). We will show below how their equivalence follows via the Poisson summation formula. We won't do all the correct analysis here [4, 3].

The Greens function must be equal to $W_{t}$ plus some homogeneous solution to the wave equation. In order to match the boundary conditions, we must choose this homogeneous solution to be the infinite array of image points $\left(W_{t}\right.$ itself provides the single source point lying within $\Omega$ ), giving

$$
G(x, y, t)=\sum_{n \in \mathbb{Z}^{d}} W_{t}(x-y-2 \pi n)
$$

Alternatively, in any compact domain $\Omega$ the Laplacian has a discrete spectrum $\left\{E_{j}\right\}, j=1,2, \ldots$ accumulating only at infinity, with corresponding orthonormal eigenmodes defined by

$$
-\Delta \phi_{j}=E_{j} \phi_{j}
$$

where $E_{j}:=k_{j}^{2}$ where $k_{j} \geq 0$ is the eigenwavenumber. At each $t$ the solution may be expressed

$$
u(x, t)=\sum_{j=1}^{\infty} a_{j}(t) \phi_{j}(x) .
$$


Inserting into (11) gives a second-order ODE for each $j$, so, matching initial conditions,

$$
a_{j}(t)=\hat{f}_{j} \cos k_{j} t+\hat{g}_{j} \frac{\sin k_{j} t}{k_{j}}
$$

analogous to (10); again the limit $k_{j} \rightarrow 0$ should be taken whenever $k_{j}=0$. Here $\hat{f}_{j}=\left\langle\phi_{j}, f\right\rangle$ are the mode coefficients of $f$, etc. Substituting these coefficients and writing out the inner product gives

$$
u(x, t)=\int_{\Omega} \sum_{j}\left(\cos k_{j} t\right) \phi_{j}(x) \overline{\phi_{j}(y)} f(y) d y+\int_{\Omega} \sum_{j} \frac{\sin k_{j} t}{k_{j}} \phi_{j}(x) \overline{\phi_{j}(y)} g(y) d y
$$

Comparing to (15) we see the Greens function is a spectral sum

$$
G(x, y, t)=\sum_{j} \frac{\sin k_{j} t}{k_{j}} \phi_{j}(x) \overline{\phi_{j}(y)}
$$

For the torus the wavevectors lie on a lattice, which motivates using the following.

Theorem 1 (Poisson summation formula). Given dimension $d \geq 1$, and $L>0$, and a smooth function $f$ which decays faster than any power of distance from the origin, i.e. $f \in \mathcal{S}\left(\mathbb{R}^{d}\right)$ a Schwarz function, then,

$$
\sum_{n \in \mathbb{Z}^{d}} f(L n)=\frac{1}{L^{d}} \sum_{m \in \mathbb{Z}^{d}} \hat{f}\left(\frac{2 \pi}{L} m\right) .
$$

Proof. The proof (from D. Bump notes) relies on realizing that the following left-hand side is a periodic function, which therefore has a Fourier series thus,

$$
\sum_{n \in \mathbb{Z}^{d}} f(x+L n)=\sum_{m \in \mathbb{Z}^{d}} a_{m} e^{-i m \cdot x}
$$

We find the $j^{\text {th }}$ coefficient by multiplying by $e^{i j \cdot x}$ and integrating over the unit cell $\Omega=[0, L)^{d}$,

$$
\sum_{n \in \mathbb{Z}^{d}} \int_{\Omega} f(x+L n) e^{i j \cdot x} d x=\sum_{m \in \mathbb{Z}^{d}} a_{m} \int_{\Omega} e^{i(j-m) \cdot x} d x
$$

The sum in the left hand side tiles the whole of space, so the expression is just $\hat{f}_{j}$. The only integral which survives in the right hand side is when $m=j$, with the value $L^{d}$. Thus we have $a_{j}=\hat{f}_{j} / L^{d}$ and the theorem is proved by setting $x=0$.

A corollary is the delta comb written as an exponential sum,

$$
\sum_{n \in \mathbb{Z}^{d}} e^{i y \cdot n}=(2 \pi)^{d} \sum_{m \in \mathbb{Z}^{d}} \delta(y-2 \pi m)
$$


in the sense of distributions, which results from the choice $f(x)=e^{-i y \cdot x}$ and $L=1$.

Returning to the torus, it is convenient to label the spectrum via $j \in \mathbb{Z}^{d}$, for which the modes are $\phi_{j}(x)=\frac{1}{(2 \pi)^{d / 2}} e^{i j \cdot x}$ with eigenvalues $E_{j}=|j|^{2}$; here $|\cdot|$ indicates the $l^{2}$-norm. The wavevectors $j$ lie on the integer lattice. Thus (26) gives

$$
G(x, y, t)=\frac{1}{(2 \pi)^{d}} \sum_{j \in \mathbb{Z}^{d}} \frac{\sin |j| t}{|j|} e^{i j \cdot(x-y)}
$$

Firstly as a special case consider $y=x$, i.e. the returning amplitude to the point $x$ after time $t$. It is tempting to apply Poisson summation directly using $f=W_{t}$ and $L=2 \pi$, thus

$$
\begin{aligned}
G(x, x, t) & =\frac{1}{(2 \pi)^{d}} \sum_{j \in \mathbb{Z}^{d}} \frac{\sin |j| t}{|j|} \\
& =\sum_{n \in \mathbb{Z}^{d}} W_{t}(2 \pi n)
\end{aligned}
$$

This is the correct expression; it agrees with (21). However, the function $W_{t}$ is not smooth, so the Fourier coefficients do not decay fast enough - this is evident as the sum over $j$ is not even absolutely convergent for $d=1$ and becomes worse for higher $d$. Thus Thm. 1 cannot be applied directly. In order to analyse such lattice sums rigorously, the sum over $j$ must be regularized. Ways to do this include i) an exponentially-decaying term, which makes the sum convergent and then taking the limit of infinite decay length [4, and ii) integrating with respect to $t$ over a Schwartz test function which has the effect of killing the sum again superalgebraically for large $|j|[3]$.

Note for all $d$ we still have $\int_{\Omega} W_{t} d x=t$. This follows from the presence of a zero-frequency mode.

\subsection{D Dirichlet box}

If instead $\Omega=[0,2 \pi)$ with Dirichlet boundary conditions the modes are given by $k_{j}=j / 2$, and $\phi_{j}(x)=\frac{1}{\sqrt{\pi}} \sin (j x / 2)$, for $j=1,2, \ldots$ It is simplest to consider the time-derivative of Greens function

$$
\begin{aligned}
\partial_{t} G(x, x, t) & =\frac{1}{2 \pi} \sum_{j}[1-\cos j x] \cos \frac{j t}{2} \\
& =\sum_{n \in \mathbb{Z}}\left[\delta(t-4 \pi n)-\frac{1}{2} \delta(t-4 \pi n-2 x)-\frac{1}{2} \delta(t-4 \pi n+2 x)\right]
\end{aligned}
$$

This can be interpreted as the repeated boundary reflections (including signchanges) of the two delta functions launched from a displacement source. The Greens function itself is a periodic square-wave-like signal.

Note the integral $\int_{\Omega} W_{t} d x=t$ now breaks down beyond the first reflection. This can be interpreted as the lack of a zero-frequency mode. 


\subsection{General domains}

In $2 \mathrm{D}$ there are other domains (namely the tiling triangles) for which the image sources (and also the eigenwavevectors) lie on a lattice, thus may handled as the torus.

The Selberg trace formula exists for certain negatively curved manifolds. I don't know whether anyone has deduced things about $G(x, y, t)$ on such manifolds.

In more general cases a modal sum is all that can be done analytically.

\section{Helmholtz equation}

We use tilde to indicate a Fourier transform from time to frequency, which follows the one-dimensional version of the convention (8). Given a frequency $\omega$, the Helmholtz equation fundamental solution $W_{\omega}$ is defined distributionally in $\mathbb{R}^{d}$ by

$$
\left(-\Delta_{x}-\omega^{2}\right) W_{\omega}(x)=\delta(x)
$$

More generally if we have a domain $\Omega$ with Dirichlet boundary conditions then the domain Greens function is

$$
\begin{array}{rlrl}
\left(-\Delta_{x}-\omega^{2}\right) G_{\omega}(x, y) & =\delta(x-y), & & x, y \in \Omega \\
G_{\omega}(x, y) & =0, & x, y \in \partial \Omega
\end{array}
$$

One can check that the above definitions of $G_{\omega}$ (or the free-space case $W_{\omega}$ ) are obtained by Fourier transforming in time the definition of the wave equation Greens function (7). Thus we can get Helmholtz Greens functions by Fourier transforming in time the above wave equation Greens functions.

\subsection{Free space}

$d=3$ We use notation $r:=|x|$. The fact that the $t^{-1}$ behavior in $W_{t}$ in $3 \mathrm{D}$ can be written instead as $r^{-1}$, therefore $t$-independent, makes for a quick result:

$$
\begin{aligned}
W_{\omega}(x) & =\int_{-\infty}^{\infty} W_{t}(x) e^{i \omega t} d t \\
& =\frac{e^{i \omega r}}{4 \pi r}
\end{aligned}
$$

$d=2$ Now there is a $t^{-1 / 2}$ tail in $W_{t}(x)$ for fixed $r$, but by changing variable 
$t=r \cosh \theta$ the integral is a standard form for the Hankel function,

$$
\begin{aligned}
W_{\omega}(x) & =\int_{r}^{\infty} \frac{e^{i \omega t}}{2 \pi \sqrt{t^{2}-r^{2}}} d t \\
& =\frac{1}{2 \pi} \int_{0}^{\infty} e^{i \omega r \cosh \theta} d \theta \\
& =\frac{i}{4} H_{0}^{(1)}(\omega r)
\end{aligned}
$$

The integral forms of Bessel functions are in Abramowitz and Stegun 9.1.23.

$d=1$ The tail in $W_{t}(x)$ for fixed $x$ now does not decay, so the integral does not converge. It only has meaning if we take the limit of a positive vanishing imaginary part to $\omega$ in which case the boundary term at $\infty$ vanishes:

$$
\begin{aligned}
W_{\omega}(x) & =\frac{1}{2} \int_{r}^{\infty} e^{i \omega t} d t \\
& =\frac{e^{i \omega r}}{2 i \omega}
\end{aligned}
$$

This is the correct form for the outgoing 1D Greens function. The convergence of the integral is not yet clear to me.

Notice as $d=1,2,3, \ldots$ we have the power of $\omega$ in front of the oscillation being $-1,-1 / 2,0, \ldots$, and the power of $r$ being $0,-1 / 2,-1, \ldots$.

\subsection{Semiclassical limit in $d=2$}

The Helmholtz fundamental solution $W_{\omega}(r)$ given by (42) is a Hankel function and we may use its well-known large-argument limit [7]

$$
\begin{aligned}
\frac{i}{4} H_{0}^{(1)}(\omega r)=\frac{i}{4} \sqrt{\frac{2}{\pi \omega r}}\left[1-\frac{8 i}{\omega r}-\right. & \frac{9}{128(\omega r)^{2}}+\cdots \\
& \left.+\frac{[(2 n-1) ! !]^{2}}{(8 i)^{n} n !} \frac{1}{(\omega r)^{n}}+\cdots\right] e^{i(\omega r-\pi / 4)}
\end{aligned}
$$

It is common in physics to use only the first term (Boasman, Smilansky, Gutzwiller, etc). We wish to understand the time-domain fundamental solution corresponding to this approximation. We will show that the above asymptotic series corresponds, term by term, to the following expansion of the time-domain fundamental solution $W_{t}(r)$, where we use $\tau=t-r$,

$$
\begin{aligned}
\frac{1}{2 \pi \sqrt{t^{2}-r^{2}}} & =\frac{1}{2 \pi \sqrt{\tau(2 r+\tau)}}=\frac{1}{2 \pi \sqrt{2 r}} \tau^{-1 / 2}\left(1+\frac{\tau}{2 r}\right)^{-1 / 2} \\
= & \frac{1}{2 \pi \sqrt{2 r}}\left(\tau^{-1 / 2}-\frac{1}{4 r} \tau^{1 / 2}+\cdots+\frac{\Gamma\left(n+\frac{1}{2}\right)}{\Gamma\left(\frac{1}{2}\right) \Gamma(n)} \frac{\tau^{n-1 / 2}}{(-2 r)^{n}}+\cdots\right)
\end{aligned}
$$


We consider only the nonvanishing region $\tau>0$. Note this is the Frobenius series (with respect to $t$ ) of $W_{t}(r)$ about the singular point (first arrival time) $t=r$. This is a Taylor series offset by the factor $(t-r)^{-1 / 2}$ which accounts for all nonanalyticity there.

To show the correspondence we first need the Fourier transform of a powerlaw on the positive real line, in the sense of distributions. By substituting $\omega=i \alpha$ and rotating the contour by $\pi / 2$ we have, for any real $p>-1$,

$$
\int_{0}^{\infty} \tau^{p} e^{i \omega \tau} d \tau=\left(\frac{i}{\omega}\right)^{p+1} \Gamma(p+1)
$$

which we may interpret as the function $H(\tau) \tau^{p}$ being the $p^{t h}$ fractional integral of $\delta(\tau)$. The time shift from $t$ to $\tau$ then introduces a factor $e^{i \omega r}$. So the Fourier transform the $n^{\text {th }}$ term (including prefactors) in (46) is

$$
\frac{(-1)^{n} i^{n+1 / 2} \Gamma\left(n+\frac{1}{2}\right)^{2}}{(\omega r)^{p+1 / 2}(2 \pi)^{p+3 / 2} p !}
$$

which, using the property $\Gamma\left(n+\frac{1}{2}\right)=\sqrt{\pi}(2 n+1) ! ! / 2^{n}$ for integer $n$, matches the $n^{\text {th }}$ term (including prefactors) in (45). This is essentially the same as the manipulation carried out to derive (45) for general order in Chapter VII.6.2 of [6].

Thus keeping only the first term in (45) corresponds to assuming precisely a $(t-r)^{-1 / 2}$ singularity in the time-domain, rather than the true form $\left(t^{2}-r^{2}\right)^{-1 / 2}$. Their relative difference only becomes large at large times $t \gg r$.

\section{Acknowledgments}

Thanks to Peter Doyle and Matt Mahoney.

\section{References}

[1] S. Levandovsky's online notes at http://www.stanford.edu/class/math220a/lecturenotes.html

[2] G. V. Nessi online notes at http://www.pdehunter.net/MathStuff/PDENotes/pdenotes.html

[3] N. Ortner, P. Wagner, "Series, the convergence of which should be interpreted in the sense of L. Schwarz", SIAM Review, 37, 428-435 (1995).

[4] D. Borwein and J. M. Borwein, "Some trigonometric and exponential lattice sums", J. Math. Anal. Appl., 188 209-218 (1994).

[5] M. E. Taylor, "Wave Equations and Diffraction", in Elsevier Encyclopedia of Mathematical Physics (2006).

[6] Courant and Hilbert, Methods of Mathematical Physics, volume 1 (1966).

[7] Abramowitz and Stegun, Handbook of Mathematical Functions. 